\title{
Eine Reihenmesskammer im Weltraum
}

Mit der Shuttle-Mission 9 hat das europäische Weltraumlabor SPACELAB erstmals die Erde umkreist. Dieser gemeinsamen NASA/ESA Mission (Tab. 1) war viel Erfolg beschieden, und sie hat deutlich gezeigt, wie unumgänglich das direkte Eingreifen von Astronauten bei Experimenten oder gar Produktionsabläufen unter Weltraumbedingungen ist. Als Beispiel sei hier nur das Experiment mit der-Reihenmesskammer (Metric Camera Experiment) erwähnt. Mit dieser Präzisionskamera (Tab. 2) wurden während dem 10tägigen Aufenthalt im Weltraum an die 1000 hochauflösende Aufnahmen nach programmiertem Plan geschossen, teils in Farbe, teils in Schwarzweiss. Trotz eines gravierenden Defektes, einer verklemmten Filmspule, konnten 90 \% des mitgeführten Filmmaterials belichtet werden. Dank genauen Anweisungen der Experimentleitung über Funk gelang es den Payload-Spezialisten die Kamera wieder in Gang zu bringen.

Die Reihenmesskammer wurde von der DFVLR (Deutsche Forschungs- und Versuchsanstalt für Luft- und Raumfahrt) in Zusammenarbeit mit der deutschen Industrie entwickelt. Planung und Bau des SPACELAB, Integration der Experimente und Durchführung der Mission erfolgte durch die ESA (European Space Agency), der Europäischen Weltraumorganisation.

Ziel des Reihenmesskammer-Experimentes ist, abzuklären, welche Möglichkeiten Aufnahmen aus niedrigen Umlaufbahnen $(250 \mathrm{~km})$ für zivile Zwecke, z.B. die Photogrammetrie, bieten. Die rund 100 ausgewählten Experimentatoren in aller Welt werden sich in den nächsten Monaten intensiv mit dem Bildmaterial befassen.

Von besonderem Interesse sind:

- die Verwendbarkeit zur Herstellung von topographischen und thematischen Karten von Gebieten, die noch nicht kartiert worden sind,

- die Verwendbarkeit zur Revision bestehender topographischer und thematischer Karten,

- die Verwendbarkeit in Verbindung mit anderen Fernerkundungsdaten, z.B. zur Verbesserung der räumlichen Auflösung,
- die Detailerkennbarkeit und radiometrische Auflösung, sowie die Präzision der Bildgeometrie ( $x, y$ und $z$ Koordinaten).

Die Forschungsergebnisse sollen in einem Symposium Ende Oktober 1984 diskutiert und von der ESA publiziert werden. Man plant heute schon einen weiteren Testflug, der im Zusammenhang mit der Earth Observation Mission-l der NASA im Frühsommer 85 stattfinden soll. Für die Zukunft werden auch folgende Verbesserungen der Kamera ins Auge gefasst:

- Kompensation der Bewegungsunschärfe

- Ausbau der Mechanik und Elektronik für die Verwendung auf einem unbemannten Satelliten

Der grosse Vorteil der Photographie aus dem Weltraum im Vergleich zu den abtastenden Sensoren in Satelliten wie NOAA, LANDSAT und SPOT, liegt in der höheren räumlichen Auflösungsleistung und den geringeren Verzerrungen in der Abbildungsgeometrie. Da ihre Auswertung zudem weniger an technische Hilfsmittel (Computer) gebunden ist, eignet sie sich besonders für den Einsatz in Ländern der 3. Welt.

Schon eine erste Durchsicht der 992 von der Metric Camera aufgenommenen Bilder lässt das Herz jedes Geowissenschafters höher schlagen. Da sind ca. $190 \mathrm{~km}$ breite Aufnahmestreifen von Schwarzafrika, von der Sahara, den Wüsten Arabiens und des Iran bis hinein nach Afghanistan. Südamerika verbirgt sich leider grösstenteils unter Wolken. Ueber Europa und den USA haben wir dank wetterglück, trotz dem wohl ungünstigsten Flugtermin bezüglich Sonnenstand, gutes Bildmaterial (siehe Titelbild). Der wohl schönste Aufnahmestreifen (in Farbe) stammt von einem Flug über die indische Tiefebene, den Ganges, über den Mt. Everest im Himalaja, das tibetanische plateau mit

Jürg Lichtenegger, Dr., ESA/EARTHNET, ESRIN, C.P. 64, I-00044 Frascati/Italia 
den geheimnisvollen Seen, bis hin zur Grenze der Mongolei. Das Gebiet war wolkenfrei und erlaubt dem mit einem stereoskop ausgerüsteten Betrachter einen ungetrübten Einblick in die landschaftlichen Schönheiten.

Fig. 1 zeigt eine mit der Metric Camera gemachte Aufnahme (Tab. 3) des Kantons Graubünden und seiner angrenzenden Gebiete. Im Gegensatz zu den Karten ist diese Aufnahme nicht nach Norden ausgerichtet. Der kurz vor dem Aufnahmedatum gefallene Schnee konnte sich wegen eines Kaltluftsees auf der Alpennordseite bis in die Niederungen halten, während auf der Alpensüdseite die Schneegrenze bei Locarno auf $1900 \mathrm{~m} / \mathrm{M}$ liegen dürfte. Ueber dem Mittelland erkennt man eine Nebeldecke, deren Obergrenze bei $1000 \mathrm{~m} / \mathrm{M}$ liegt. Man beachte unter anderem die

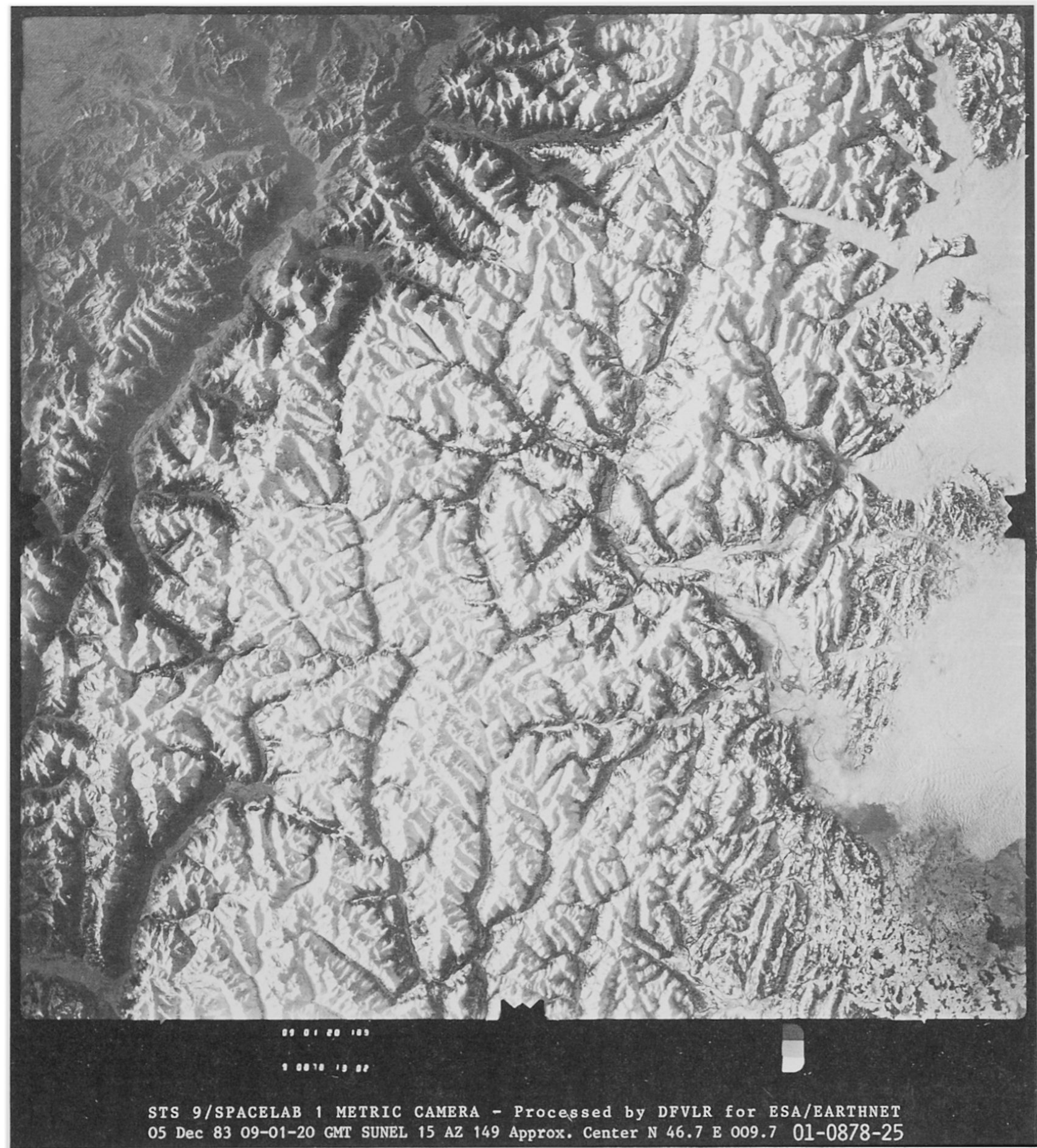

Fig. 1: Aufnahme der Metric Camera (Reihenmesskammer), Originalbild, der östlichen Alpen aus $250 \mathrm{~km}$ Höhe am 5. Dez. 83, 10 Uhr. Bildidentifikation 01-0878-25. 
Wellenstruktur auf der Nebeloberseite in der Gegend der Linthebene und des Bodensees. Da über Mitteleuropa eine windschwache Hochdrucklage herrschte, kann angenommen werden, dass Kaltluft, der Schwere folgend, aus dem grossen Einzugs- Linsen: gebiet des Rheins abfloss, sich adiabatisch erwärmte und über den Kaltluftsee des Mittellandes schob. An der Grenzschicht entstand dadurch Turbulenz. Die Talenge von Ziegelbrücke erzeugte zudem eine Düsenwirkung.

Das Titelbild ist eine Ausschnittsvergrösserung. Es zeigt das Rheintal von Reichenau über Chur bis Sargans. Der Kontrast wird durch den Schnee noch erhöht und lässt auch Flurwege und einzelne Häuser erkennen. Selbst in Schattenpartien (Sonnenelevation nur 19 Grad) können Strassen, Siedlungen und wälder kartiert werden.

Kopien aller Bilder der Metric Camera sind erhältlich auf Film und Papier in Formaten $\mathrm{zwischen} 25 \times 25$ und $50 \times$ $60 \mathrm{~cm} \mathrm{zu}$ Preisen von DM 37.-- bis DM 330.--. Bestellungen nimmt entgegen: DFVLR, WT-DA FE, D-8031 Wessling/BRD.
Tabelle 2:

Merkmale der SPACELAB Reihenmesskammer

Typ:

Kalibrierte

Brennweite:

max. Verzeichnung:

Auflösung:

Oeffnungswinkel:

modifizierte Zeiss

RMK A $30 / 23$

Topar Al mit

7 Linsenelementen

$305.128 \mathrm{~mm}$

$6 \mu \mathrm{m}$ (gemessen)

$39 \mathrm{LP} / \mathrm{mm}$ AWAR auf

Aviphot PAN $30 \mathrm{Film}$

diagonal $56^{\circ}$, über

die seite $41.2^{\circ}$

Verschluss:

Aerotop Rotations-

lamellenverschluss

Filmplanlage:

durch Gebläsemotor

im Kameragehäuse

Verschlusszeiten: $1 / 250$ bis $1 / 1000$ sec

in 31 schritten

Blende:

5.6 bis 11.0 in 31

Schritten

Belichtungsfre-

quenz

Bildformat:

4 bis 6 sec und

8 bis 12 sec

$23 \times 23 \mathrm{~cm}$

$24 \mathrm{~cm}$

$150 \mathrm{~m}=550$ Einzelbilder

Filmlänge:

Abmessung Kamera: $46 \times \begin{array}{lllll}4 & x & x & 52 & \mathrm{~cm}\end{array}$

Masse Kamera:

$54.0 \mathrm{~kg}$

$24.5 \mathrm{~kg} \mathrm{mit}$ Film

Eine gute Uebersicht bieten 8 Microfiches, auf denen sämtliche Aufnahmen abgebildet sind. Ebenfalls erhältlich ist eine Liste mit den geographischen Koordinaten der Bildmittelpunkte mit Angaben über die Wolkenbedeckung und weiteren Spezifikationen. Kostenpunkt: rund SFr. 75.--. Bestellungen und weitere Fragen sind $\mathrm{zu}$ richten an: ESA/EARTHNET, C.P. 64, I-00044 Frascati/Italia, Tel. 39(6)9401l.

Tabelle I:

Merkmale der Mission STS9/SPACELAB

$\begin{array}{ll}\text { Abschuss: } & 28 . \mathrm{Nov} .1983 \\ \text { Landung: } & 8 . \mathrm{Dez} 1983 \\ \text { Flughöhe: } & 240-257 \mathrm{~km} \\ \text { Inklination: } & 570 \\ \text { Geschwindigkeit: } & 7.7 \mathrm{~km} / \mathrm{sec} \\ \text { Bildmasstab: } & 1: 820000 \\ \text { Bedeckung eines } & \\ \text { Bildes: } & 189 \times 189 \mathrm{~km} \\ \text { Bildwanderung: } & \text { bei } 1 / 500 \mathrm{sec} 18 \mathrm{~mm} \\ & \text { oder } 16 \mathrm{~m} \text { am Boden } \\ \text { Film: } & \text { Kodak Double-X Aero- } \\ & \text { graphic Film 2405 } \\ & \text { Kodak Aerochrome } \\ & \text { Infrared Film } 2443 \\ \text { Bildmaterial: } & 543 \text { Falschfarbenaufn. } \\ & 449 \text { Schwarzweissaufn. }\end{array}$

Tabelle 3:Merkmale der Aufnahme Fig. I

Bildindentifikation: 01-0878-25

Aufnahmedatum: 5. Dez. 1983

Aufnahmezeit: $\quad 9$ Uhr $01 \mathrm{~min}$

Flughöhe:

Blende:

20 sec $G M T$

Verschlusszeit

$250 \mathrm{~km}$

5.7

Ueberlappung:

$1 / 550 \sec$

Bildzentrum:

Sonnenelevation:

$80 \%$

$46.7 N \quad 9.7 \quad E$

Sonnenazimut:

Film:

$19^{\circ}$

$149^{\circ}$

Kodak Double-X

Aerographic Film

2405 (Schwarzweiss) 